\title{
Clinical phenotype of germline RUNX1 haploinsufficiency: from point mutations to large genomic deletions
}

\author{
Mylène Béri-Dexheimer ${ }^{1}$, Véronique Latger-Cannard ${ }^{2}$, Christophe Philippe ${ }^{1}$, \\ Céline Bonnet ${ }^{1}$, Pascal Chambon ${ }^{1}$, Virginie Roth ${ }^{1}$, Marie-José Grégoire ${ }^{1}$, Pierre Bordigoni ${ }^{3}$, \\ Thomas Lecompte $^{2}$, Bruno Leheup ${ }^{4}$ and Philippe Jonveaux ${ }^{\star 1}$
}

\footnotetext{
${ }^{1}$ Laboratoire de Génétique, Centre Hospitalier Universitaire de Nancy Brabois, Nancy Université, EA4002, France; ${ }^{2}$ INSERM U734, Laboratoire d'Hématologie, Nancy Université, CHU Nancy, France; ${ }^{3}$ Service de Médecine Infantile 1, CHU Nancy, France; ${ }^{4}$ Service de Médecine Infantile 3 et de Génétique Clinique, CHU Nancy, France
}

Germline RUNX1 mutations result in a rare autosomal dominant condition characterized by qualitative and quantitative platelet defects and predisposition to the development of myeloid malignancies (familial platelet disorder with propensity to acute myeloid leukaemia, FPD/AML). Only 13 pedigrees have previously been described so far. We report on two novel germline RUNX1 mutations: (1) an out-of-frame 8 bp heterozygous deletion (c.442_449del) in an FPD/AML pedigree and (2) a de novo $3.5 \mathrm{Mb}$ deletion in the $21 \mathrm{q} 22.11 .21 \mathrm{q} 22.12$ region encompassing the $R U N X 1$ gene in a mentally retarded female patient with short stature and thrombocytopenia. Interestingly, a similar de novo submicroscopic deletion has been recently reported in the literature in a mentally retarded patient. Mental retardation is one of the most common disorders and primary causes of thrombocytopenia are rare. When occurring together, these features should prompt to test for 21 q22 deletion for comprehensive genetic counselling and clinical management.

European Journal of Human Genetics (2008) 16, 1014-1018; doi:10.1038/ejhg.2008.89; published online 14 May 2008

Keywords: RUNX1; inherited thrombocytopenia; 21q22 microdeletion

\section{Introduction}

Familial platelet disorder with propensity to acute myeloid leukaemia (FPD/AML; OMIM 601399) is an autosomal dominant disorder in which affected members have a clinical history of bleeding tendency and mild to moderate thrombocytopenia with normal platelet size and morphology, and/or abnormal platelet aggregation in response to arachidonic acid. ${ }^{1}$ Predisposition to develop myelodysplasia

*Correspondence: Professor Dr P Jonveaux, Laboratoire de Génétique, EA 4002, Centre Hospitalier Universitaire de Nancy Brabois, Nancy Université, Rue du Morvan, Vandoeuvre les Nancy F-545111, France. Tel: + 333831537 71; Fax: + 333831537 72;

E-mail: p.jonveaux@chu-nancy.fr

Received 4 December 2007; revised 6 March 2008; accepted 11 March 2008; published online 14 May 2008 and AML is another feature of this platelet disorder, with a leukaemic rate of approximately $35 \%$. Affected individuals within the same family may present with variable clinical severity at varying ages. The disorder is located on chromosome 21q22.12, and germline heterozygous mutations in the haematopoietic transcription factor $R U N X 1$ (also known as AML1 and CBFA2) have been identified in 13 pedigrees reported so far, including missense, frameshift, nonsense mutations and a large intragenic deletion. ${ }^{2-7}$ In the present study, we describe two novel germline RUNX1 mutations including a large genomic deletion influencing the clinical phenotype. We therefore suggest giving consideration to cryptic deletion of chromosome $21 \mathrm{q} 22.11 \mathrm{q} 22.12$ in the diagnosis of unexplained mental retardation/thrombocytopenia syndromes. 


\section{Methods and results}

Blood samples and clinical information from all patients analysed were obtained with approval of the institutional review boards of Nancy University hospital.

\section{Case 1}

The male proband was first seen in 1999 at the age of 2 because of purpura without history of easy bruising and bleeding. Physical examination did not reveal any abnormal findings except ecchymoses and petechiae. His blood count showed haemoglobin $13.4 \mathrm{~g}$ per $100 \mathrm{ml}$, normal leucocyte count $5.67 \times 10^{9} / 1$ and decreased platelet count $50 \times 10^{9} / 1$. Peripheral blood smear examination showed platelet of normal size and morphology. Coagulations tests (APTT, PT and fibrinogen) were in normal ranges. Platelet studies showed the absence of second-wave aggregation in response to adenosine diphosphate (ADP, $10 \mu \mathrm{M})$ and a reduced and reversible response to collagen $(2 \mu \mathrm{g} / \mathrm{ml})$ and arachidonic acid $(1.39 \mathrm{mM})$. ATP secretion from dense granules in response to collagen $(20 \mu \mathrm{g} / \mathrm{ml})$ and thrombin receptor agonist peptide (TRAP) was markedly reduced respectively (peak ATP concentration proband: $0.8 \mu \mathrm{M}$ ATP/healthy donor: $13 \mu \mathrm{M}$ ATP; proband: $1.3 \mu \mathrm{M}$ ATP/healthy donor: $11.8 \mu \mathrm{M}$ ATP). Flow cytometry analysis showed a reduced uptake and release of mepacrine, a compound selectively taken up by dense granules, ${ }^{8,9}$ associated with a low expression of CD63, a membrane marker of dense granule, on activated platelets. These abnormalities were in favour of platelet dense granule disorder or $\delta$-storage pool disease $(\delta$-SPD). Membrane glycoprotein expression, GPIIb/IIIa (CD41a) and GMP140
(CD62P) was normal before and after TRAP stimulation. $\mathrm{GPIb} \alpha$ (CD42b) was weakly internalized after platelet stimulation. After 4 years, at the age of 6 , he developed AML. Peripheral blood analysis showed pancytopenia with haemoglobin $5.8 \mathrm{~g}$ per $100 \mathrm{ml}$, WBC $2.1 \times 10^{9} / \mathrm{l}$ and a platelet count of $10 \times 10^{9} / 1$. A bone marrow aspirate yielded a very hypercellular bone marrow with blasts (20\%) and myelodysplastic features. Blasts phenotype was immature with myeloid markers leading to AML diagnosis. Cultured leukaemic cells showed a normal karyotype. He underwent successful bone marrow transplantation from HLA-haploidentical donor and is still in remission 4 years later.

His mother, a woman without any bleeding or physical abnormality, was found to have mild isolated thrombocytopenia $\left(120-150 \times 10^{9} / 1\right)$ and abnormal platelet behaviour consistent with a $\delta$-SPD. Haematological features of the proband's maternal grandparents were normal. To further elucidate the genetic origin of this autosomal dominant platelet disorder, exons 3-6 of the RUNX1 gene were amplified from genomic DNA extracted from peripheral blood samples of the male proband, his father and his mother. Primer pairs were designed as previously described. ${ }^{2}$ The purified PCR products were bidirectionally sequenced with the BigDye Terminator 1.1 Cycle Sequencing Kit (Applied Biosystems). An out-of-frame $8 \mathrm{bp}$ heterozygous deletion (c.442_449del) was identified in exon 4 of RUNX1 in the genomic DNA of the proband and his mother (Figure 1a). Sequence variation identified in exon 4 of RUNX1 was numbered starting from the first base of the ATG start codon, numbering based on reference

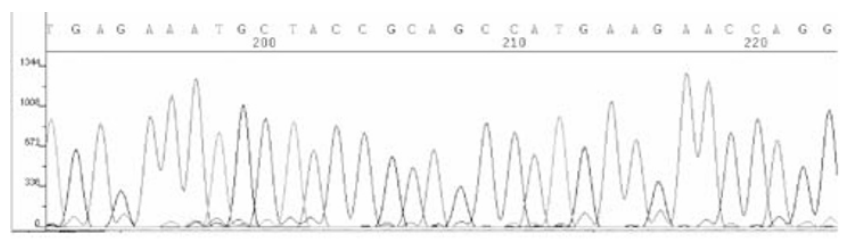

TGAGAAATGCTACCGCAGCCATGAAGAACCAG

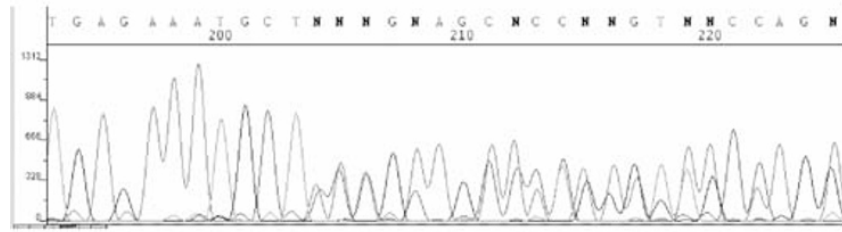

TGAGAAATGCTACCGCAGCCATGAAGAACCAG

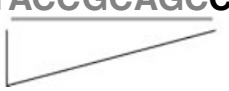

TGAGAAATGCTCATGAAGAACCAGGTTGCAAG b

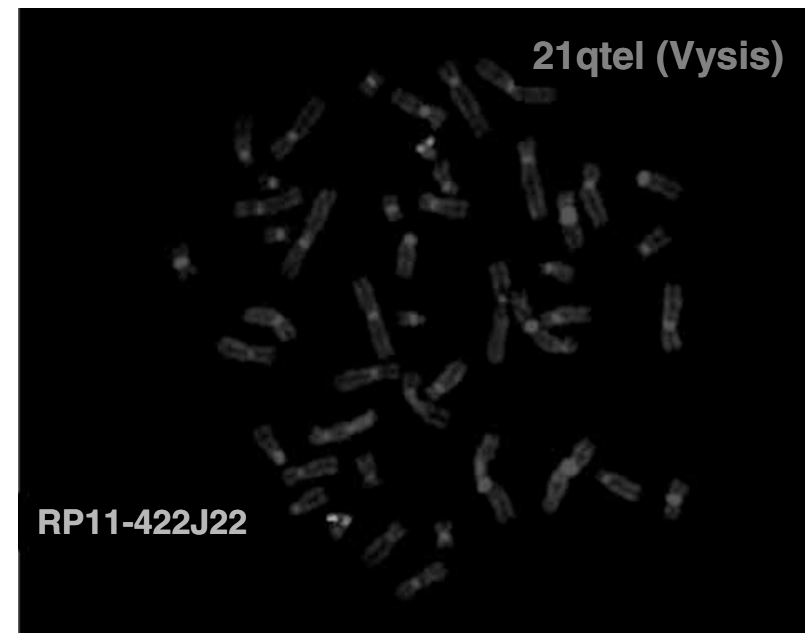

Figure 1 (a) DNA sequence of the Case 1 patient (bottom) showing the 8 bp deletion in exon 4 of $R U N X 1$, and a control sequence (top). (b) FISH analysis of the clone RP11-422J22 (34958334-35122861) showing only one green signal on metaphase chromosome 21 . The red signal corresponds to the control probe on 21 qter (Vysis Inc.). 
sequence NM_001754.3. The effect of the $8 \mathrm{bp}$ deletion at the protein level was predicted with the EMBOSS Transeq software (http://www.ebi.ac.uk/). If translated, the mutated allele would lead to a severely truncated protein (p.Thr148HisfsX9). However, the mutant mRNA harbours a premature termination codon (PTC), such PTC often triggers nonsense-mediated mRNA decay of mRNA. ${ }^{10}$ Although the mutant mRNA level has not been studied in vitro, this out-of-frame deletion most probably corresponds to a null allele.

\section{Case 2}

This girl was first seen at the age of 10 to explore speech and developmental delay associated with short stature and microcephaly. She is the only child of unrelated healthy parents (a 19-year-old father and a 16-year-old mother at the conception). Family history was unremarkable. During gestation growth delay was reported at the fifth-month ultrasonographic examination. At the same time multiple renal cysts were also seen in the left kidney. Cytogenetic analysis on amniotic fluid was normal. She was born at full term with low weight $(-3 \mathrm{SD})$, short length $(-3.5 \mathrm{SD})$ and microcephaly $(-3 \mathrm{SD})$. Hypotonia was present. A severe inter-atrial septal defect due to hypoplastic septum primum required surgical correction at 1 year of age. The genital examination showed hypoplastic labia majora. Micropolygyria of the right frontal and parietal area was reported by MRI. At the age of 10, clinical evaluation revealed height at $-2.5 \mathrm{SD}$, with persistent microcephaly (-2.3 SD). Craniofacial dysmorphism included a prominent metopic ridge, upslanted palpebral fissures, anteverted nares, moderate telecanthus and downturned corners of the mouth with thin lips. Neurodevelopmental features included psychomotor retardation with severely delayed receptive and expressive language skills. She had not any history of bleeding. Peripheral blood analysis showed isolated thrombocytopenia (platelet count of $\left.57 \times 10^{9} / 1\right)$ with a normal platelet morphology. Platelet studies revealed reduced and reversible response to ADP $(10 \mu \mathrm{M})$ and arachidonic acid $(1.39 \mathrm{mM})$. ATP secretion in response to collagen $(20 \mu \mathrm{g} / \mathrm{ml})$ and TRAP was markedly reduced (peak ATP concentration proband: $0.8 \mu \mathrm{M} /$ healthy donor: $10.1 \mu \mathrm{M}$; proband: $0.8 \mu \mathrm{M}$ /healthy donor: $10.4 \mu \mathrm{M})$. Flow cytometry analysis of dense granules showed a reduced uptake and release of mepacrine associated with a low expression of CD63. These abnormalities were in favour of $\delta$-SPD diagnosis. Membrane glycoprotein expression GPIb $\alpha$ (CD42b) and GMP140 (CD62P) was normal before and after TRAP stimulation. GPIIb/IIIa (CD41a) expression was weakly reduced on resting and stimulated platelet. A DNA sample from the patient was analysed using the GenoSensor Array (Vysis Inc., Abbott Laboratories SA, Downers Grove, IL, USA) according to the protocols recommended by the manufacturer and as described previously. ${ }^{11}$ This array contained 287 genomic targets cloned from six PAC and BAC libraries, unevenly distributed over the genome, including cancer amplicons/ oncogenes, tumour-suppressor genes, loci of insertions/ deletions/duplications, telomeres and markers added to reduce gaps (http://www.vysis.com). The microarray slides were analysed using the GenoSensor Reader System (Vysis Inc.) according to the manufacturer's instructions. On the basis of previous control experiments using the GenoSensor Array 300, we determined losses and gains of target DNA sequence copy number by a green/red ratio $<0.8$ and $>1.2$ respectively. A deletion of a $21 \mathrm{q} 22$ clone (RP11-422J22) including RUNX1 was detected. To confirm and further characterize the deletion, FISH analysis was performed using BAC probes (The Wellcome Trust Sanger Institute, UK) mapping to 21q22.11q22.12 (Figure 1b). According to the UCSC genome browser (March 2006), the proximal breakpoint mapped between clone RP11-430F9 and RP11-165-F7 whereas the distal breakpoint resided between clone RP11-73C15 and RP11-135F21 leading to a deleted region of approximately $3.3 \mathrm{Mb}$ including 29 confirmed and predicted genes. The karyotype is written as: 46,XX.arr cgh 21q22.12(RUNX1)x1.ish del(21) (q22.11q22.12)(RP11-165F7-,RP11-67E8-,RP11-79D9-,RP11357B7-,RP11-133J19-,RP11-415I16-,RP11-422J22-,RP11-451M12-, RP11-118P22-,RP11-475I12-,RP11-73C15-). Maternal 21q22.12 FISH using the RP11-422J22 probe encompassing the RUNX1 gene was normal. The father was not available for investigation.

\section{Discussion}

Heterozygous germline mutation in the RUNX1 gene (UCSC browser, March 2006: 21q22. 12: 3508196835182857) causes FPD with predisposition to acute myelogenous leukaemia. FPD is not fatal but significantly inhibits blood clotting, which could be life threatening after surgery, injury or dental treatment. The incidence of leukaemia among affected individuals varies from 20 to $50 \%$. The affected subjects seem to develop myeloid malignancies throughout their lifespan. FDP/AML is extremely rare worldwide with a total of 13 pedigrees identified so far. In addition to a novel two-generation family with typical FDP/AML and a frameshift RUNX1 mutation, we described in a mentally retarded patient with short stature and thrombocytopenia, a microdeletion that includes RUNX1 and several neighbouring genes. Larger chromosome deletions spanning the $21 \mathrm{q} 22$ region have previously been reported in patients with mental impairment and multiple abnormalities, ${ }^{12-13}$ unfortunately without any accurate size. To the best of our knowledge, only one similar microdeletion has been recently identified during a study of 100 children with idiopathic mental retardation and normal standard chromosomal analysis, using a GeneChip mapping 100K SNP array. ${ }^{14}$ Only an 


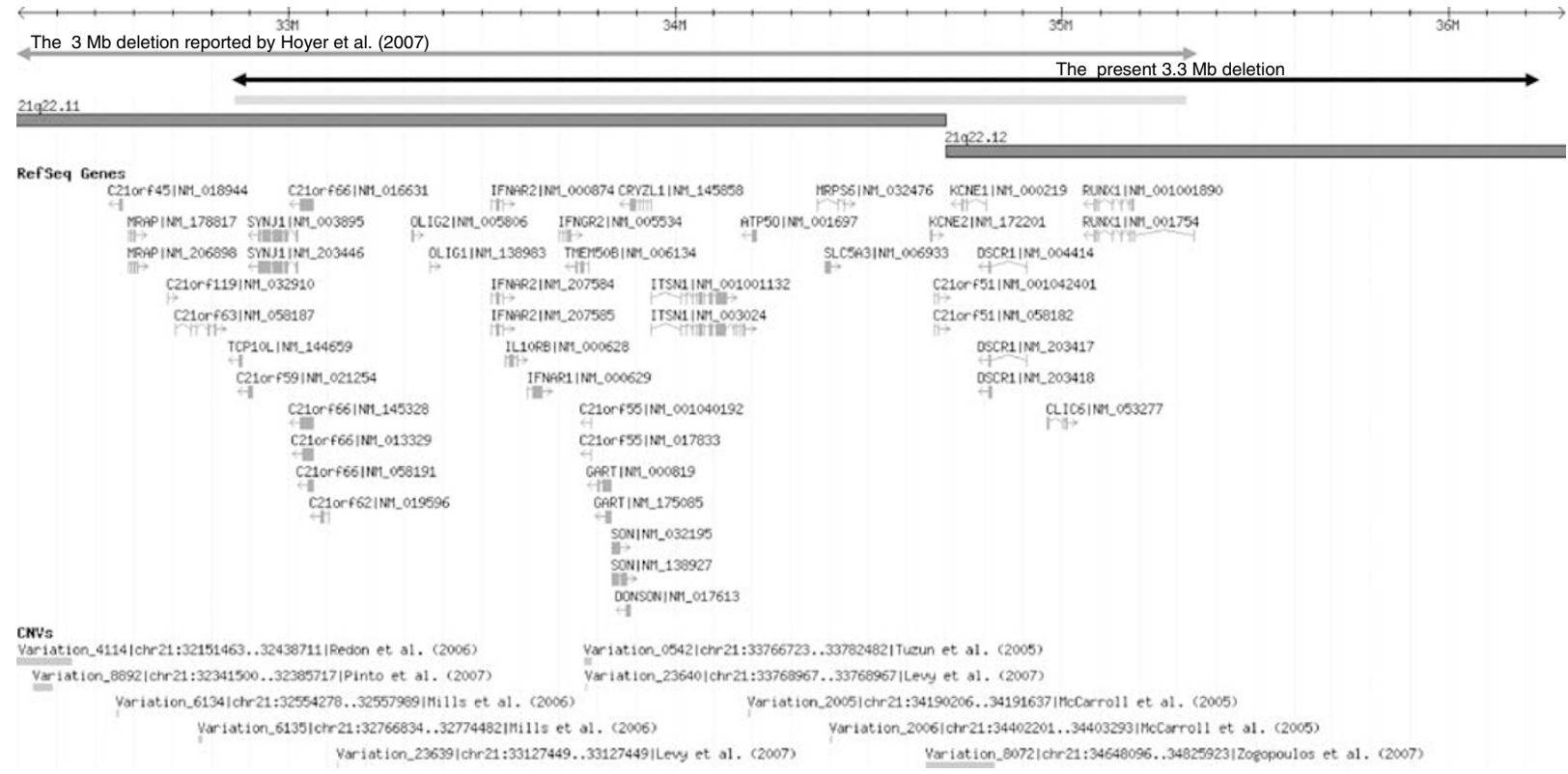

Figure 2 Map of the $21 \mathrm{q} 22.11 \mathrm{q} 22.12$ region showing the relative position of Refseq genes and genomic variants. The grey line indicates the extent of the $3 \mathrm{Mb}$ deletion reported by Hoyer et al, ${ }^{14}$ the black line indicates the extent of the $3.3 \mathrm{Mb}$ deletion identified in the present patient and the blue line indicates the overlapping aberrant region. The drawing is based on the UCSC map (March 2006) and the Database of Genomic Variants.

overview of the clinical findings was reported. This patient had a short stature without major morphological anomalies. Breakpoints were mapped from SNP rs8129832 (32329260) to SNP rs10483024 (35362570) indicating a $3 \mathrm{Mb}$ deletion. It would be interesting to look for a platelet disorder in this patient as the deletion includes the RUNX1 gene. Actually, this deletion shares 25 out of 29 genes included in our patient's deletion (Figure 2). Furthermore, the author confirmed the de novo occurrence of the deletion, strengthening a disease-causing deletion in our patient. No evidence of segmental duplications or lowcopy repeats was found near any of the four breakpoints in these two patients. According to the Database of Genomic Variants, a curated catalogue of large-scale variation in the human genome (http://projects.tcag.ca/variation/), some copy number polymorphisms have been described on 21q22.11q22.12. These data can aid in the identification of potential genes that are more likely to be causative of the patients' phenotype. In addition to $R U N X 1$ haploinsufficiency clearly involved in FDP/AML, at least four candidate genes, SYNJ1, ITSNL1, RCAN1 and CLIC6, located outside polymorphic regions, could contribute to mental retardation. Synaptojanin-1 is a nerve terminal protein that appears to participate with dynamin in synaptic vesicle recycling. ${ }^{15}$ Intersectin 1 is a cytoplasmic membraneassociated protein that indirectly coordinates endocytic membrane traffic with the actin assembly machinery. In addition, the encoded protein may regulate the formation of clathrin-coated vesicles and could be involved in synaptic vesicle recycling. ${ }^{16}$ The Down's syndrome critical region protein RCAN1 regulates long-term potentiation and memory by inhibition of phosphatase signalling. ${ }^{17}$ CLIC6, a member of the intracellular chloride channel family, interacts with dopamine $\mathrm{D}(2)$-like receptors. ${ }^{18}$

Finally, inherited thrombocytopenia associated with mental retardation is an uncommon condition. The Jacobsen syndrome (OMIM 147791) with the Paris-Trousseau syndrome (OMIM 188025), the thrombocytopeniaabsent radius (TAR) syndrome (OMIM 274000) and the 22q11.2 deletion syndrome (OMIM 192430) have additional distinct clinical anomalies. Patients with Jacobsen syndrome have trigonocephaly, facial dysmorphism, cardiac defects and Paris-Trousseau-type platelet defects, ie mild thrombocytopenia with a subpopulation of platelets containing giant $\alpha$-granules. TAR syndrome is characterized by severe isolated thrombocytopenia at birth that spontaneously improves after the first year of life. The absence of radii with retention of normal thumbs is characteristic and can be associated with renal and cardiac defects. In the chromosome 22q11.2 deletion syndrome, mental retardation can be associated with thrombocytopenia with large platelets in addition to conotruncal cardiac anomalies, facial dysmorphism and immunodeficiency. On the basis of these observations, 21q22.11q22.12 microdeletion could be a novel cause of mental retardation, in which a platelet disorder (thrombocytopenia and/ or abnormal platelet aggregation) would be a distinctive phenotypic manifestation. 


\section{Acknowledgements}

This work was supported by the regional PHRC 2002 of the Ministère de la Santé. We thank the Cytogenetics staff at the Nancy University hospital for their expert technical assistance.

\section{References}

1 Ho CY, Otterud B, Legare RD et al: Linkage of a familial platelet disorder with a propensity to develop myeloid malignancies to human chromosome 21q22.1-22.2. Blood 1996; 87: 5218-5224.

2 Song WJ, Sullivan MG, Legare RD et al: Haploinsufficiency of CBFA2 causes familial thrombocytopenia with propensity to develop acute myelogenous leukaemia. Nat Genet 1999; 23: 166-175.

3 Buijs A, Poddighe P, van Wijk R et al: A novel CBFA2 singlenucleotide mutation in familial platelet disorder with propensity to develop myeloid malignancies. Blood 2001; 98: 2856-2858

4 Michaud J, Wu F, Osato $\mathrm{M}$ et al: In vitro analyses of known and novel RUNX1/AML1 mutations in dominant familial platelet disorder with predisposition to acute myelogenous leukemia: implications for mechanisms of pathogenesis. Blood 2002; 99: $1364-1372$.

5 Walker LC, Stevens J, Campbell $\mathrm{H}$ et al: A novel inherited mutation of the transcription factor RUNX1 causes thrombocytopenia and may predispose to acute myeloid leukaemia. Br I Haematol 2002; 117: 878-881.

6 Sun L, Mao G, Rao AK: Association of CBFA2 mutation with decreased platelet PKC-theta and impaired receptor-mediated activation of GPIIb-IIIa and pleckstrin phosphorylation: proteins regulated by CBFA2 play a role in GPIIb-IIIa activation. Blood 2004; 103: 948-954.

7 Heller PG, Glembotsky AC, Gandhi MJ et al: Low Mpl receptor expression in a pedigree with familial platelet disorder with predisposition to acute myelogenous leukemia and a novel AML1 mutation. Blood 2005; 105: 4664-4670.
8 Gordon N, Thom J, Cole C, Baker R: Rapid detection of hereditary and acquired platelet storage pool deficiency by flow cytometry. Br J Haematol 1995; 89: 117-123.

9 Wall J, Buijs-Wilts M, Arnold J et al: A flow cytometry assay using mepacrine for study of uptake and release of platelet dense granule contents. Br J Haematol 1995; 89: 380-385.

10 Maquat LE: Nonsense-mediated mRNA decay: splicing, translation and mRNP dynamics. Nat Rev Mol Cell Biol 2004; 5: 89-99.

11 Korshunov A, Sycheva R, Golanov A: Genetically distinct and clinically relevant subtypes of glioblastoma defined by arraybased comparative genomic hybridization. Acta Neuropathol 2006; 111: $465-474$.

12 Chettouh Z, Croquette MF, Delobel B et al: Molecular mapping of 21 features associated with partial monosomy 21: involvement of the APP-SOD1 region. Am J Hum Genet 1995; 57: 62-71.

13 Theodoropoulos DS, Cowan JM, Elias ER, Cole C: Physical findings in $21 \mathrm{q} 22$ deletion suggest critical region for $21 \mathrm{q}$ phenotype in q22. Am J Med Genet 1995; 59: 161-163.

14 Hoyer J, Dreweke A, Becker C et al: Molecular karyotyping in patients with mental retardation using $100 \mathrm{~K}$ single-nucleotide polymorphism arrays. J Med Genet 2007; 44: 629-636.

15 Lee SY, Wenk MR, Kim Y et al: Regulation of synaptojanin 1 by cyclin-dependent kinase 5 at synapses. Proc Natl Acad Sci USA 2004; 101: 546-551.

16 Evergren $\mathrm{E}$, Gad $\mathrm{H}$, Walther $\mathrm{K}$ et al: Intersectin is a negative regulator of dynamin recruitment to the synaptic endocytic zone in the central synapse. J Neurosci 2007; 27: 379-390.

17 Hoeffer CA, Dey A, Sachan N et al: The Down syndrome critical region protein RCAN1 regulates long-term potentiation and memory via inhibition of phosphatase signaling. I Neurosci 2007; 28: $13161-13172$.

18 Griffon N, Jeanneteau F, Prieur F et al: CLIC6 a member of the intracellular chloride channel family, interacts with dopamine D(2)-like receptors. Brain Res Mol Brain Res 2003; 117: $47-57$. 